\title{
EA-Matrix integrals of associative algebras and equivariant localization.
}

\section{Serguei Barannikov}

To R.K.Gordin on the occasion of his 70th birthday

Keywords Mirror symmetry, Gromov-Witten invariants, Noncommutative varieties, Batalin-Vilkovisky formalism Abstract The EA-matrix integrals, introduced in [B1], are studied in the case of graded associative algebras with odd or even scalar product. I prove that the EA-matrix integrals for associative algebras with scalar product are integrals of equivariantly closed differential forms with respect to Lie algebra $g l_{N}(A)$.

\section{Introduction}

The theory of periods of noncommutative varieties, depending on commutative parameters, was introduced in [B5]. The analogue of top-degree holomorphic form in this setting was shown in loc.cit. to be certain element of semi-infinite subspace of negative cyclic homology. The integrals of this element satisfy the second order equation with respect to the parameters of deformations of the varieties. It was proven in loc.cit. that the generating function of genus zero GromovWitten invariants of complete intersection in $\mathbb{C P}^{d}$ with trivial canonical class coincides with the coefficient of this second order equation for the family of mirror varieties. This approach had singled out the $A_{\infty}-$ algebras/categories, satisfying cyclic homology analogue of degeneration of Hodge to de Rham spectral sequence, as the proper definition of (smooth and compact) noncommutative varieties.

The EA-matrix integrals were introduced in [B1] as a set of periods of associative, more generally $A_{\infty}-$ algebras or noncommutative varieties, depending on noncommutative parameters:

$$
\mathcal{F}(Y)=\int_{\Gamma} \exp \left(\operatorname{Tr}\langle Y, X\rangle+\frac{1}{3 !} m_{\tilde{A}}\left(X, \frac{\partial}{\partial X}\right)\right) \vdash \prod_{\alpha, i, j} d X_{i}^{\alpha, j}
$$

$\tilde{A}=A \otimes q_{N} / \tilde{A}=A \otimes g l_{N}$ in even/odd scalar product case, here $q_{N}$ is the odd matrix algebra, see loc.cit. It was shown in theorem 3 in loc.cit. that the matrix Airy integral from [K] corresponds in this way to the simplest associative algebra of one dimension $A=\left\{e \mid e^{2}=e\right\}$.

The usual varieties correspond here to $A_{\infty}$ - algebras of endomorphisms of generators of their $D^{b}($ Coh) - categories.

The asymptotic expansion of EA-matrix integrals via BV formalism was shown in [B1, B3] to define, as a sum over generalized ribbon graphs, a generating function for series of cohomology classes of compactified moduli spaces of curves of all genus. A particular example is the formula for cohomology-valued generating function for products of $\psi$-classes, $\psi_{i}=c_{1}\left(T_{p_{i}}^{*}\right)$, in the cohomology $H^{*}\left(\overline{\mathcal{M}}_{g, n}\right)$ calculated by the stable ribbon graph complex $([\mathrm{B} 3])$ :

$$
\sum_{\sum d_{i}=d} \psi_{1}^{d_{1}} \ldots \psi_{n}^{d_{n}} \prod_{i=1}^{n} \frac{\left(2 d_{i}-1\right) ! !}{\lambda_{i}^{\left(2 d_{i}+1\right)}}=\left[\sum_{G \in \Gamma_{g, n}^{d e c, o d d}} G \frac{2^{-\chi(G)}}{|\operatorname{Aut}(G)|} \prod_{e \in \operatorname{Edge}(G)} \frac{1}{\lambda_{i(e)}+\lambda_{j(e)}}\right]
$$

Preprint CNRS HAL-00507788 (2010)

IMJ-UMR7586 CNRS, l'Université de Paris; NRU-HSE

E-mail: serguei.barannikov@imj-prg.fr 
where the sum on the right is over stable ribbon oriented graphs of genus $g$ with $n$ numbered punctures, with $2 d+n$ edges, and such that at each vertex the cyclically ordered subsets of edges have arbitrary odd cardinality.

In this paper it is proven that the EA-matrix integrals for associative algebras with scalar product are integrals of equivariantly closed differential forms with respect to the Lie algebra $g l_{N}(A)$. This generalizes and clarifies the similar result with respect to the Lie algebra $g l_{N}$ from [B2]. The localization formula for the $g l_{N}(A)$-action then leads in [B4] to calculation of these EA matrix integrals via determinants and $\tau$-functions of integrable hierarchies.

The paper is dedicated to Raphail Kalmanovich Gordin, with gratitude.

\section{Acknowledgements}

The results of this paper were presented starting from 2010 at conferences in Miami, Vienna, Tokyo, Moscow, Bonn, Boston. I am thankful to organizers of these conferences for their hospitality and for the opportunity to present these results to large audience, and to participants for interesting questions.

\section{Notations}

For a $\mathbb{Z} / 2 \mathbb{Z}$-graded vector space $A=A_{0} \oplus A_{1}$ denote via $\Pi A$ the parity inverted vector space, $(\Pi A)_{0}=A_{1},(\Pi A)_{1}=$ $A_{0}$. For an element $a$ from $\mathbb{Z} / 2 \mathbb{Z}$-graded vector space $A$ denote by $\pi a \in \Pi A$ the same element considered with parity reversed.

\section{Equivariantly closed De Rham differential form.}

Let $A=A_{0} \oplus A_{1}$ denotes a $\mathbb{Z} / 2 \mathbb{Z}$-graded associative algebra, $\operatorname{dim}_{k} A_{0}=r<\infty, \operatorname{char}(k)=0$, with multiplication denoted by $m_{2}: A^{\otimes 2} \rightarrow A$. Let $A$ be endowed with odd invariant non-degenerate scalar product $\langle\cdot, \cdot\rangle: A_{0} \otimes A_{1} \rightarrow k$. The multiplication tensor can be viewed then as the $\mathbb{Z} / 3 \mathbb{Z}$ - cyclically invariant linear function on $(\Pi A)^{\otimes 3}$

$$
m_{A}:\left(\pi a_{1}, \pi a_{2}, \pi a_{3}\right) \mapsto(-1)^{\bar{a}_{2}+1}\left\langle m_{2}\left(a_{1}, a_{2}\right), a_{3}\right\rangle, m_{A} \in\left(\operatorname{Hom}\left((\Pi A)^{\otimes 3}, k\right)\right)^{\mathbb{Z} / 3 \mathbb{Z}} .
$$

The odd symmetric scalar product on $A$ corresponds to the odd anti-symmetric product $\langle\cdot, \cdot\rangle^{\pi}$ on $\Pi A$ :

$$
\left\langle\pi a_{1}, \pi a_{2}\right\rangle^{\pi}=(-1)^{\bar{a}_{1}+1}\left\langle a_{1}, a_{2}\right\rangle
$$

The tensor product with the matrix algebra $g l_{N}$ is again naturally a $\mathbb{Z} / 2 \mathbb{Z}$ - graded associative algebra with the odd scalar product $A \otimes g l_{N}$. The cyclic tensor

$$
m_{A \otimes g l_{N}} \in\left(\operatorname{Hom}\left(\left(\Pi A \otimes g l_{N}\right)^{\otimes 3}, k\right)\right)^{\mathbb{Z} / 3 \mathbb{Z}}
$$

restricted to the diagonal $\Pi A \otimes g l_{N} \subset\left(\Pi A \otimes g l_{N}\right)^{\otimes 3}$ is $G L(N)$-invariant cubic polynomial, denoted by $m_{A \otimes g l_{N}}(Z)$, $Z \in \Pi A \otimes g l_{N}$. The associativity of the algebra $A$ translates into the equation

$$
\left\{m_{A \otimes g l_{N}}(Z), m_{A \otimes g l_{N}}(Z)\right\}=0
$$

where $\{\cdot, \cdot\}$ is the odd Poisson bracket corresponding to the odd anti-symmetric product $\left.\operatorname{Tr}\right|_{g l_{N}^{\otimes 2}} \otimes\langle\cdot, \cdot\rangle^{\pi}$ on $\Pi A \otimes g l_{N}$.

Proposition 1 The algebra of functions on $\Pi A \otimes g l_{N}$ is identified naturally, preserving the odd Poisson bracket, with the algebra of polyvectors on the even affine space $\Pi A_{1} \otimes g l_{N}$. 
This is analogous to algebra of functions on symplectic space being identified naturally, preserving Poisson bracket, with algebra of functions on cotangent bundle of given lagrangian subspace.

Denote by $X^{\alpha} \in g l_{N}, P_{\alpha} \in \Pi g l_{N}$ the matrices of coordinates on $\Pi A \otimes g l_{N}$ corresponding to a choice of a dual pair of bases $\left\{e^{\alpha}\right\},\left\{\xi_{\alpha}\right\}$ on $A_{0}$ and $A_{1}$ so that

$$
Z=\sum_{\alpha} \pi \xi_{\alpha} \otimes X^{\alpha}+\pi e^{\alpha} \otimes P_{\alpha}
$$

Then $\left(P_{\alpha}\right)_{j}^{i}$ corresponds to the vector field $\frac{\partial}{\partial\left(X^{\alpha}\right)_{i}^{j}}$ on $\Pi A_{1} \otimes g l_{N}$. The cubic polynomial $\frac{1}{3 !} m_{A \otimes g l_{N}}(Z)$ corresponds to the sum of the function and the bivector,

$$
\frac{1}{3 !} \sum_{\alpha, \beta, \gamma}\left(m_{A}\right)_{\alpha \beta \gamma} \operatorname{Tr}\left(X^{\alpha} X^{\beta} X^{\gamma}\right)+\frac{1}{2} \sum_{\alpha, \beta, \gamma}\left(m_{A}\right)_{\alpha}^{\beta \gamma} \operatorname{Tr}\left(X^{\alpha} P_{\beta} P_{\gamma}\right)
$$

The odd Poisson bracket is generated by the odd second order Batalin-Vilkovisky differential $\Delta$ acting on the algebra of functions on $\Pi A \otimes g l_{N}$

$$
\begin{gathered}
\left\{f_{1}, f_{2}\right\}=(-1)^{\bar{f}_{1}}\left(\Delta\left(f_{1} f_{2}\right)-\Delta\left(f_{1}\right) f_{2}+(-1)^{\bar{f}_{1}} f_{1} \Delta\left(f_{2}\right)\right) \\
\Delta=\sum_{\alpha, i, j} \frac{\partial^{2}}{\partial X_{i}^{\alpha, j} \partial P_{\alpha, j}^{i}}
\end{gathered}
$$

1.1 Divergence-free condition

Let us assume from now on that the Lie algebra $A_{0}$ is unimodular.

Condition 2 (unimodularity of $A_{0}$ ) For any $a \in A_{0}$

$$
\operatorname{tr}\left(\left.[a, \cdot]\right|_{A_{0}}\right)=0
$$

Proposition 3 The unimodularity of $A_{0}$ (1.6) implies

$$
\Delta m_{A \otimes g l_{N}}(Z)=0 .
$$

Next proposition is the standard corollary of the equations (1.2), (1.7) and the relation (1.5).

Proposition 4 The exponent of the sum (1.4) is closed under the Batalin-Vilkovisky differential

$$
\Delta \exp \left(\frac{1}{3 !} m_{A \otimes g l_{N}}(Z)\right)=0 .
$$

\subsection{Closed De Rham differential form}

The affine space $\Pi A_{1} \otimes g l_{N}$ has a holomorphic volume element, defined canonically up to a multiplication by a constant

$$
\varpi=\lambda \prod_{\alpha, i, j} d X_{i}^{\alpha, j} .
$$

It identifies the polyvectorfields on $\Pi A_{1} \otimes g l_{N}$ with the de Rham differential forms $\Omega_{\Pi A_{1} \otimes g l_{N}}$ on the same affine space via

$$
\gamma \mapsto \gamma \vdash \varpi
$$


The Batalin-Vilkovisky differential $\Delta$ corresponds then to the De Rham differential $d_{D R}$ acting on the differential forms. By the proposition 4 the polyvector $\exp \frac{1}{3 !}\left(m_{A \otimes g l_{N}}(Z)\right)$ defines the closed differential form

$$
\begin{gathered}
\Psi(X)=\exp \left(\frac{1}{3 !} \sum_{\alpha, \beta, \gamma}\left(m_{A}\right)_{\alpha \beta \gamma} \operatorname{Tr}\left(X^{\alpha} X^{\beta} X^{\gamma}\right)+\frac{1}{2} \sum_{\alpha, \beta, \gamma}\left(m_{A}\right)_{\alpha}^{\beta \gamma} \operatorname{Tr}\left(X^{\alpha} \frac{\partial}{\partial X^{\beta}} \wedge \frac{\partial}{\partial X^{\gamma}}\right)\right) \vdash \lambda \prod_{\alpha, i, j} d X_{i}^{\alpha, j} \\
d_{D R} \Psi(X)=0
\end{gathered}
$$

It is a sum of the closed differential forms of degrees $r N^{2}, r N^{2}-2, \ldots$

1.3 Equivariantly closed differential form

The unimodularity (1.6) implies the invariance of $\varpi$ under the co-adjoint action of the Lie algebra $A_{0} \otimes g l_{N}$

$$
X \mapsto[Y, X]
$$

$Y \in A_{0} \otimes g l_{N}$. Consider the $A_{0} \otimes g l_{N}$-equivariant differential forms on $\Pi A_{1} \otimes g l_{N}$ :

$$
\Omega_{\Pi A_{1} \otimes g l_{N}}^{A_{0} \otimes l_{N}}=\left(\Omega_{\Pi A_{1} \otimes g l_{N}} \otimes \mathcal{O}_{A_{0} \otimes g l_{N}}\right)^{A_{0} \otimes g l_{N}} .
$$

The $A_{0} \otimes g l_{N}$-equivariant differential is given by

$$
d_{A_{0} \otimes g l_{N}} \Phi(Y)=d_{D R} \Phi-\sum_{\alpha, l, j} Y_{a, j}^{l}\left(i_{\left[E_{l}^{j} \otimes e^{a}, \cdot\right]} \Phi\right)
$$

$\Phi \in \Omega_{\Pi A_{1} \otimes g l_{N}}^{A_{0} \otimes g l_{N}}$, where $i_{\gamma}$ denotes the contraction operator with respect to the vector field $\gamma$, see e.g. [DKV]. This differential corresponds, when passing to functions on $\Pi A \otimes g l_{N}$, to the sum

$$
\begin{aligned}
\Delta_{A_{0} \otimes g l_{N}} & : f(Z, Y) \mapsto \Delta f-\frac{1}{2} \operatorname{Tr}\langle[Y, Z], Z\rangle^{\pi} f, \\
f(Z, Y) & \in\left(\mathcal{O}_{\Pi A \otimes g l_{N}} \otimes \mathcal{O}_{A_{0} \otimes g l_{N}}\right)^{A_{0} \otimes g l_{N}}
\end{aligned}
$$

of the Batalin-Vilkovisky differential and the operator of multiplication by the odd quadratic function

$$
\frac{1}{2} \operatorname{Tr}\langle[Y, Z], Z\rangle^{\pi}=m_{A \otimes g l_{N}}(Y \otimes Z \otimes Z) .
$$

The function depends on the equivariant parameters $Y \in A_{0} \otimes g l_{N}$.

Theorem 1 The product of the closed de Rham differential form $\Psi(X)$ (1.8) with the function $\exp \operatorname{Tr}\langle Y, X\rangle, Y \in$ $A_{0} \otimes g l_{N}, X \in \Pi A_{1} \otimes g l_{N}$, is $A_{0} \otimes g l_{N}$-equivariantly closed differential form:

$$
d_{A_{0} \otimes g l_{N}}\left(\exp \left(T r\langle Y, X\rangle+\frac{1}{3 !} m_{A \otimes g l_{N}}\left(X, \frac{\partial}{\partial X}\right)\right) \vdash \lambda \prod_{\alpha, i, j} d X_{i}^{\alpha, j}\right)=0
$$

Proof Denote by $i_{m\left(X \frac{\partial}{\partial X} \frac{\partial}{\partial X}\right)}$ the operator of contraction with the bivector field $\frac{1}{2} \sum_{\alpha, \beta, \gamma}\left(m_{A}\right)_{\alpha}^{\beta \gamma} \operatorname{Tr}\left(X^{\alpha} \frac{\partial}{\partial X^{\beta}} \wedge \frac{\partial}{\partial X^{\gamma}}\right)$ and by $R_{\operatorname{Tr}(Y d X)}$ the operator of exterior multiplication by the 1-form $\operatorname{Tr}\langle Y, d X\rangle$ acting on differential forms,

$$
R_{T r(Y d X)}=\left[d_{D R}, i_{T r\langle Y, X\rangle}\right]
$$

where $i_{\operatorname{Tr}\langle Y, X\rangle}$ is the multiplication by the linear function $\operatorname{Tr}\langle Y, X\rangle$. Then

$$
\left[i_{m\left(X \frac{\partial}{\partial X} \frac{\partial}{\partial X}\right)}, R_{\operatorname{Tr}(Y d X)}\right]=i_{[\cdot, Y]}
$$

This is simply a particular case of the standard relation

$$
\left[i_{\gamma_{1}}, \operatorname{Lie}_{\gamma_{2}}\right]=i_{\left[\gamma_{1}, \gamma_{2}\right]}
$$


for the action of polyvector fields. Notice that

$$
d_{D R} e^{T r\langle Y, X\rangle}=e^{\operatorname{Tr}\langle Y, X\rangle}\left(d_{D R}+R_{T r}(Y d X)\right)
$$

and that

$$
R_{\operatorname{Tr}(Y d X)} \exp \left(i_{m\left(X \frac{\partial}{\partial X} \frac{\partial}{\partial X}\right)}\right)=\exp \left(i_{m\left(X \frac{\partial}{\partial X} \frac{\partial}{\partial X}\right)}\right)\left(R_{\operatorname{Tr}(Y d X)}+i_{[\cdot, Y]}\right)
$$

Since $d_{D R} \Psi(X)=0$, and $R_{\operatorname{Tr}(Y d X)} \prod_{\alpha, i, j} d X_{i}^{\alpha, j}=0$, therefore

$$
d_{D R}\left(e^{T r\langle Y, X\rangle} \Psi(X)\right)=i_{[\cdot, Y]} e^{T r\langle Y, X\rangle} \Psi(X) .
$$

\section{The integral.}

The closed differential form $\Psi(X)$ is integrated over the cycles, which are standard in the theory of exponential integrals $\int_{\Gamma} \exp f$, see $(\underline{\mathrm{AVG}}]$ and references therein):

$$
\Gamma \in H_{*}(M, \operatorname{Re}(f) \rightarrow-\infty), M=\Pi A_{1} \otimes g l_{N}(\mathbb{C})
$$

Here $f$ is the first term in (1.4), which is the restriction of the cubic polynomial $\frac{1}{3 !} m_{A \otimes g l_{N}}(Z)$ on $M$.

The relative homology are the same for such $f, f \neq 0$, and for $f+\operatorname{Tr}\langle Y, X\rangle$ since linear term is dominated by the cubic term when $|X| \rightarrow+\infty$. Choosing a real form of $A_{0} \otimes g l_{N}(\mathbb{C})$ and taking the cycles in $H_{*}(M, \operatorname{Re}(f) \rightarrow-\infty)$ invariant with respect to this Lie algebra gives natural cycles for integration of the equivariantly closed differential form $e^{\operatorname{Tr}\langle Y, X\rangle} \Psi(X)$

$$
\mathcal{F}(Y)=\int_{\Gamma} \exp \left(T r\langle Y, X\rangle+\frac{1}{3 !} m_{A \otimes g l_{N}}\left(X, \frac{\partial}{\partial X}\right)\right) \vdash \prod_{\alpha, i, j} d X_{i}^{\alpha, j}
$$

In general the integration cycles are the elements of the equivariant homology

$$
H_{*, A_{0} \otimes g l_{N}}\left(M, \operatorname{Re}\left(\frac{1}{3 !} \sum_{\alpha, \beta, \gamma}\left(m_{A}\right)_{\alpha \beta \gamma} \operatorname{Tr}\left(X^{\alpha} X^{\beta} X^{\gamma}\right)\right) \rightarrow-\infty\right)
$$

One can consider also the normalized integral

$$
\widehat{\mathcal{F}}(Y)=\int_{\Gamma} \exp \left(T r\langle Y, X\rangle+\frac{1}{3 !} m_{A \otimes g l_{N}}\left(X, \frac{\partial}{\partial X}\right)\right) \vdash \prod_{\alpha, i, j} d X_{i}^{\alpha, j} / \mathcal{F}_{[2]}(Y)
$$

where $\mathcal{F}_{[2]}(Y)$ is the corresponding Gaussian integral of the quadratic part of $f+\operatorname{Tr}\langle Y, X\rangle$ at a critical point $(-Y)^{\frac{1}{2}}$.

Let the associative algebra $A_{0}$ has an anti-involution $a \rightarrow a^{\dagger}$

$$
(a b)^{\dagger}=b^{\dagger} a^{\dagger},(c a)^{\dagger}=\bar{c} a^{\dagger}, \operatorname{tr}\left(a^{\dagger}\right)=\overline{\operatorname{tr}(a)},\left(a^{\dagger}\right)^{\dagger}=a
$$

The anti-involution defines the natural cycle for the equivariant integration. This anti-involution extends naturally to $A_{0} \otimes g l_{N}(\mathbb{C})$. Then the Lie subalgebra of anti-hermitian elements in $A_{0} \otimes g l_{N}(\mathbb{C})$

$$
u_{N}\left(A_{0}\right)=\left\{Y^{\dagger}=-Y \mid Y \in A_{0} \otimes g l_{N}(\mathbb{C})\right\}
$$

is a real form of $A_{0} \otimes g l_{N}(\mathbb{C})$. And the space of hermitian elements in the dual space

$$
\Gamma=\left\{X^{\dagger}=X \mid X \in A_{0}^{\vee} \otimes g l_{N}(\mathbb{C})\right\}
$$

is invariant under the action of $u_{N}\left(A_{0}\right)$. Then the "real-slice" $\Gamma$ is the natural choice of the cycle for the equivariant integration.

The localization formula for equivariant cohomology reduces the integral of the equivariantly closed form $\Omega$ over $\Gamma$ to the integral over the fixed locus $F$,

$$
\int_{\Gamma} \Omega=\int_{F} \frac{\Omega}{e u\left(N_{F}\right)}
$$


where $e u\left(N_{F}\right)$ is the euler class of the normal bundle of $F$ in $\Gamma$, see $\mathrm{AB}$, [DKV]. Calculating the integral using the equivariant localization leads to generalized Vandermond determinants and $\tau$ - functions.

Let for simplicity the algebra $A$ with odd scalar product is the tensor product $A=A_{0} \otimes q_{1}$ of the even associative algebra $A_{0}$ with scalar product, denoted $\eta\left(y_{1}, y_{2}\right)$, and the algebra $q_{1}=\left\{1, \xi \mid \xi^{2}=1\right\}$ with the odd scalar product $\langle 1, \xi\rangle=1$.

Assume that the natural scalar product on the Lie algebra of anti-hermitian elements in $A_{0}$ is positive definite

$$
-\eta(y, y)=\eta\left(y, y^{\dagger}\right)>0
$$

Otherwise one can apply to $\Gamma$ a partial Wick rotation.

Then the localization formula (2.4) after some calculations leads to the following result ([B4]):

Proposition 5 The integral (2.2), written in variables $t \in H C^{*}(A)$, is a $\tau$-function of KP-type hierarchy and, in particular, satisfies the Hirota quadratic equations.

\section{References}

AVG. V.Arnold, A.Varchenko, S.Gusejn-Zade, Singularities of Differentiable Mappings. v.1, 2. M.: Nauka, (1985)

AB. M.Atiyah, R.Bott, The moment map and equivariant cohomology, Topology 23 (1984) no. 1, 1-28.

B1. S.Barannikov, Noncommutative Batalin-Vilkovisky geometry and matrix integrals. Preprint NI06043 (2006), Cambridge University, preprint CNRS hal-00102085 (2006). Comptes Rendus Mathématique vol 348, pp. 359-362. doi: 10.1016/j.crma.2010.02.002

B2. S.Barannikov, Solving the noncommutative Batalin-Vilkovisky equation. Preprint CNRS hal-00464794 (2010). Letters in Mathematical Physics (2013) Vol 103, Issue 6, pp 605-628. doi:10.1007/s11005-013-0615-8

B3. S.Barannikov, Supersymmetry and cohomology of graph complexes. Preprint CNRS hal-00429963 (2009), Letters in Mathematical Physics (2018) doi:10.1007/s11005-018-1123-7

B4. S.Barannikov, EA-matrix integrals and cyclic cohomology. Preprint CNRS hal-02144148

B5. S.Barannikov, Quantum periods - I. Semi-infinite variations of Hodge structures. arXiv:math/0006193(2000), IMRN Vol. 2001, pp.12431264 doi:10.1155/S1073792801000599

DKV. M.Duflo, Sh.Kumar, M.Vergne, Sur la cohomologie équivariante des variétés différentiables. Astérisque 215 (1993).

K. M.Kontsevich Intersection theory on the moduli space of curves and the matrix Airy function. Comm. Math. Phys. 147 (1992), no.1, $1-23$. 